\title{
Why a Combination of WP 631 and Epo B is an Improvement on the Drugs Singly - Involvement in the Cell Cycle and Mitotic Slippage
}

\author{
Barbara Bukowska ${ }^{1 *}$, Aneta Rogalska ${ }^{1}$, Ewa Forma ${ }^{2}$, Magdalena Brys ${ }^{2}$, \\ Agnieszka Marczak ${ }^{1}$
}

\begin{abstract}
Our previous studies clearly demonstrated that a combination of WP 631 and Epo B has higher activity against ovarian cancer cells than either of these compounds used separately. In order to fully understand the exact mechanism of action in combination, we assessed effects on the cell cycle of $\mathrm{SKOV}-3$ cells. We evaluated three control points essential for WP 631 and Epo B action to determine which cell cycle-regulating proteins (CDK1/cyclin B complex, EpCAM or HMGB1) mediate activity. The effects of the drug on the cell cycle were measured based on the nuclear DNA content using flow cytometry. Expression of cell cycle-regulating genes was analyzed using real-time PCR. It was discovered that WP 631, at the tested concentration, did not affect the SKOV-3 cell cycle. Epo B caused significant G2/M arrest, whereas the drug combination induced stronger apoptosis and lower mitotic arrest than Epo B alone. This is very important information from the point of view of the fight against cancer, as, while mitotic arrest in Epo B-treated cells could be overcame after DNA damage repair, apoptosis which occurs after mitotic slippage in combination-treated cells is irreversible. It clearly explains the higher activity of the drug combination in comparison to Epo B alone. Epo B acts via the CDK1/cyclin B complex and has the ability to inhibit CDK1, which may be a promising strategy for ovarian cancer treatment in the future. The drug combination diminishes EpCAM and HMGB1 expression to a greater degree than either WP 631 and Epo B alone. Owing to the fact that the high expression of these two proteins is a poor prognostic factor for ovarian cancer, a decrease in their expression, observed in our studies, may result in improved efficacy of cancer therapy. The presented findings show that the combination of WP 631 and Epo B is a better therapeutic option than either of these drugs alone.
\end{abstract}

Keywords: Apoptosis - cell cycle - epothilone B - ovarian cancer - WP 631

Asian Pac J Cancer Prev, 17 (3), 1299-1308

\section{Introduction}

Ovarian cancer is one of the commonest causes of female cancer death, with about $75 \%$ of patients diagnosed in advanced stages. Many women with ovarian cancer develop resistance to standard chemotherapy, hence new therapeutic options are highly desirable (Arikan et al., 2014; Chen et al., 2014; Ye et al., 2014). For this reason, we decided to focus on the combined action of WP 631, a member of anthracyclines, and epothilone B (Epo B). The results of our previous study clearly demonstrate that WP 631 and Epo B synergize in ovarian cancer cells. We have already shown that the combination of WP 631 and Epo $\mathrm{B}$ induces apoptosis, leads to DNA damage and provokes oxidative stress more intensively than those compounds used separately (Marczak et al., 2014; Rogalska et al., 2014). However, the exact mechanism of action of this drug regimen remains undiscovered. Seeing that the dysregulation of the cell cycle is a hallmark of cancer cells, fully understanding the influence of anticancer drugs on the distribution of each phase of the cell cycle allows the development of new chemotherapeutic strategies and thus the improvement of clinical outcomes (Senese et al., 2014). For this reason, we decided to investigate the effect of this drug combination on the cell cycle of SKOV-3 cells.

The effect of single drugs, such as WP 631 and Epo B, on cancer cell cycle has been well established. Griffin et al. demonstrated that Epo B induced G2/M accumulation, followed by apoptosis in ovarian cancer cells (Griffin et al., 2003). Moreover, the results of Pellicciotta et al. show that Epo B leads to cell cycle arrest in the G2/M phase (Pellicciotta et al., 2013). The ability of WP 631 to block cell cycle progression in the $\mathrm{G} 2 / \mathrm{M}$ phase was confirmed in the other studies (Villamarin et al., 2003; Pozarowski et al., 2004; Mansilla et al., 2006). To the best of our knowledge, we are the first to investigate the combined 
effect of WP 631 and Epo B on the cell cycle. We focused on three control points (presented in Figure1) essential for WP 631 and Epo B activity. The choice of cell cycleregulating proteins was preceded by a literature review where it was found that the single agents may influence the cell cycle.

One of the chosen proteins was CDK1 (cyclindependent kinase 1), which controls normal cell cycle progression by promoting transition from the $\mathrm{G} 2$ to $\mathrm{M}$ phase. During the G1 phase, CDK1 remains inactive, due to low concentrations of cyclins and the high activity of cyclin dependent kinase inhibitors (CKIs) (Enserink and Kolodner, 2010). In the G2 phase, phosphorylation of CDK1 on Tyr14 and Tyr15 prevents unscheduled CDK1 activation, whereas dephosphorylation at these sites prevents the inhibition of CDK1 during early mitosis. For the full activation of CDK1, binding of cyclin B, its activating partner, and then phosphorylation on Thr161 are required (Kobayashi et al., 2014). At the end of the metaphase, APC complex (anaphase promoting complex) is responsible for cyclin B degradation. The elevated expressions of CKIs, as well as cyclin destruction, are signals for mitotic exit (Chow et al., 2011). The impact of WP 631, Epo B, and their combination on CDK1 activity remains unknown. However, paclitaxel, with a mechanism of action similar to Epo B, activates CDK1 and CDK2 in breast cancer cell lines (Nakayama et al., 2009). In contrast, doxorubicin, a widely used member of anthracyclines, combined with tamoxifen, decreases CDK1 and CDK2 in breast cancer cells (Chuang et al., 2013). Taking into account these contradictory literature data, we decided to evaluate the levels of CDK1 and its activator, cyclin B, in SKOV-3 cell line. In order to assess whether these two proteins are involved in the combined mechanism of the action of WP 631 and Epo B, the activity of CDK1/cyclin B complex was blocked by alsterpaullone. Alsterpaullone is a small molecule cyclin-dependent kinase inhibitor with a potential to stop the cell cycle and to induce apoptosis by caspase-9 activation and PARP cleavage (Cui et al., 2013). It stops the action of CDK1/ cyclin B complex by binding to the ATP-binding site of CDK1, thereby stopping cell cycle progression.

Moreover, we focused our attention on the EpCAM protein (cell adhesion molecule), surface glycoprotein, whose expression is higher in epithelial cancer, e.g. ovarian carcinoma, which correlates with poor prognosis and decreased overall survival (van der Gun et al., 2010). Activation of EpCAM may lead to upregulation of c-Myc and thus, as a result, in upregulation of cyclin A and cyclin E (Munz et al., 2004). EpCAM also indirectly affects cyclin D1 expression at the transcriptional level (ChavesPerez et al., 2013). The activity of EpCAM is controlled by regulated intramembrane proteolysis (RIP). The first step of EpCAM cleavage is catalyzed by ADAM17 protein, which leads to EpCAM's ectodomain (EpEX) release. In the second step, PS-2 ( $\gamma$-secretase) cuts off EpCAM's cytoplasmatic tail (EpICD), which combines with FHL-2 and $\beta$-catenin. Such a complex is translocated to the cell nucleus and affects the transcription of EpCAM targeted genes (Schnell et al., 2013). While Epo B upregulates EpCAM expression at nanomolar concentrations (Shahabi et al., 2010), little is known about the effect of WP 631, therefore we decided to study the influence of the tested drugs on EpCAM expression at the transcriptional level in the SKOV-3 cell line. In order to find out if EpCAM mediates the mechanism of the action of the tested drugs, its activity was blocked by DAPT (N-[N- $(3,5-$ Difluorophenacetyl)-L-alanyl]-S-phenylglycine t-butyl ester), which inhibits $\gamma$-secretase and thus prevents EpICD cutting. Its ability to inhibit EpCAM was established in colon cancer cells (Lin et al., 2012).

Another interesting target for our study was the HMGB1 protein (high-mobility group box-1) which is a nuclear protein including the RB (retinoblastoma)binding motif called LXCXE. In the studies of Jiao et al., it was determined that HMGB1 enhances RB-mediated repression of cyclin A and E2F transcription, following by suppression of cell growth, G1 arrest and apoptosis in MCF-7 breast cancer cells (Jiao et al., 2007). Although these results indicate that HMGB1 may function as a tumor suppressor, it is also responsible for ovarian cancer growth and metastasis. The HMGB1 gene knockout decreased cell proliferation and reduced the metastatic ability of ovarian cancer cells (Chen et al., 2012). The bioinformatic analysis conducted by Gong et al. also showed that overexpression of HMGB1 correlates with oncoproteins cyclin D and cyclin E overexpression, whereas tumor suppressor proteins, such as p53, are inhibited (Gong et al., 2010). Literature data indicates that anthracyclines stimulates HMGB1 release, but the impact of WP 631, belonging to bisanthracyclines, as well as Epo B and the drug combination, remains undiscovered. For this reason, we evaluated the drugs influence on HMGB1 mRNA expression. To determine if this protein is engaged in the mechanism of the action of the tested compounds, the activity of HMGB1 was blocked by metformin, biguanide class of oral hypoglycemic agents (Tsoyi et al., 2011; Zhang et al., 2014). Recent evidence pointed out that metformin may reduce the risk of cancer (Saito et al., 2013).

In Figure 1 we show the influence of the presented proteins, CDK1/cyclin B, EpCAM and HMGB1, on cell cycle progression. The role of cyclin $\mathrm{CDK} 1 / \mathrm{B}$ complex is to transit the cells from $\mathrm{G} 2$ to $\mathrm{M}$ phase. EpCAM, through the impact on cyclins A, D and E, affects G1/S and S/G2 progression. Similarly, HMGB 1 affecting cyclins D and E, regulates transition from $\mathrm{G} 1$ to $\mathrm{S}$ phase. All these proteins act at different points in the cell cycle, however, their up or downregulation may lead to serious disturbances of this cycle. Therefore, knowledge about the interconnectedness between anticancer drugs and cell cycle-regulating proteins broadens our understanding of the mechanism of the action of WP $631+$ Epo B combination in SKOV-3 ovarian cancer cells.

\section{Materials and Methods}

\section{Chemicals}

Alsterpaullone, D A P T $\quad(\mathrm{N}-[\mathrm{N}-(3,5-$ Difluorophenacetyl)-L-alanyl]-S-phenylglycine t-butyl ester), metformin, WP 631, epothilone B, ribonuclease $\mathrm{A}$, and propidium iodide (PI) were acquired from Sigma- 
Why a Combination of WP 631 and Epo B is an Improvement on the Drugs Singly - Involvement in the Cell Cycle and Mitotic Slippage

Aldrich (St. Louis, USA). Trypsin-EDTA, Fetal Bovine Serum (FBS) penicillin and streptomycin were purchased from PAA Laboratories GmbH (Pasching, Austria). High Capacity cDNA Reverse Transcription Kit was obtained from Life Technologies (Carlsbad, USA) and ExtractMe Total RNA Kit was acquired from Blirt (Gdańsk, Poland). The other chemicals and solvents, of a high analytical grade, were supplied by POCH S.A. (Gliwice, Poland).

\section{Cell culture and drug administration}

SKOV-3 (human ovarian adenocarcinoma) cells were obtained from American Type Culture Collection (ATCC, Rockville, USA). The cells were grown as a monolayer at $37{ }^{\circ} \mathrm{C}$ in a $5 \%$ carbon dioxide atmosphere and $100 \%$ humidity. RPMI: RPMI 1640 growth medium was supplemented with $10 \% \mathrm{FBS}$, penicillin $(10 \mathrm{U} / \mathrm{ml})$ and streptomycin $(50 \mu \mathrm{g} / \mathrm{ml})$. The cells were free of Mycoplasma contamination.

The tested drugs were dissolved in a suitable solvent (ethanol for Epo B and DMSO for WP 631). They were stored frozen at $-20^{\circ} \mathrm{C}$ divided into small portions (20$50 \mu \mathrm{l}$ each). Concentrated drug solutions were thawed immediately before using, and then diluted in PBS and added to the cell medium at the final concentration.

It has previously been demonstrated that WP 631 and Epo B synergize in SKOV-3 cell line. The most potent activity was obtained for the combination, in which concentrations of both tested drugs were equal (1:1 combination, $5 \mathrm{nM}$ of WP 631 and $5 \mathrm{nM}$ of Epo B) (Marczak et al., 2014). The cells were seeded into Petri dishes with a diameter of $60 \mathrm{~mm}$ at a density of $500 \times 10^{3}$ cells/Petri dish for both experiments (cell cycle analysis and real-time PCR technique). After $24 \mathrm{~h}$, in both presented methods, drugs in the following concentrations: WP 631 and Epo B separately - $10 \mathrm{nM}$, the $1: 1$ drugs combination at a final concentration of $10 \mathrm{nM}(5 \mathrm{nM}+5$ $\mathrm{nM}$ ) were added for various lengths of time (4, 16 and 24 h) into the culture conditions. As part of these experiments, an additional one hour preincubation with cell cycle inhibitors (alsterpaullone, DAPT, and metformin) was performed.

\section{Cell cycle analysis}

Cellular DNA content was quantified by flow cytometry. SKOV-3 cells were treated with drugs for 4,16 , and $24 \mathrm{~h}$. In the variant with cell cycle inhibitors, first one hour preincubation with alsterpaullone, DAPT or metformin was carried out. Then WP 631, Epo B or the WP 631 and Epo B combination at the appropriate concentration were added and incubation was continued for the required period of time under the same condition. After the incubation cells were collected, washed twice with phosphate-buffered saline (PBS) and fixed in $70 \%$ ethanol. After ethanol fixation (at least $24 \mathrm{~h}$ at $4^{\circ} \mathrm{C}$ ), the cells were washed in PBS and then centrifuged at $7000 \mathrm{~g}$ for $10 \mathrm{~min}$ at $4^{\circ} \mathrm{C}$. Pelleted cells were stained by adding $300 \mu 1$ of PBS containing PI and RNase at final concentrations of $75 \mu \mathrm{M}$ and $20 \mu \mathrm{g} / \mathrm{ml}$, respectively. Then a one-hour incubation in the total darkness at $37^{\circ} \mathrm{C}$ was performed. Stained cells were analyzed using a flow cytometer (Becton Dickinson, USA). The cell populations in particular phases of the cell cycle were quantified from a standard count of 10,000 cells by means of Flow Jo cytology software.

\section{RNA extraction and cDNA synthesis}

Total RNA was isolated from cells using ExtractMe Total RNA Kit (Blirt, Poland), according to the manufacturer's protocol. The quantity and quality of the isolated RNA were assessed spectrophotometrically. First strand cDNAs were obtained by reverse transcription of $2 \mu \mathrm{g}$ of total RNA, using High Capacity cDNA Revers Transcription Kit (Life Technology, USA), following the manufacturer's protocol. cDNAs were stored at $-20^{\circ} \mathrm{C}$.

\section{Quantitative real time PCR analysis}

The relative expression levels of HMGB1, EpCAM, CDK1 and CCNB1 genes were analyzed by real time PCR using the primer pairs HMGB 1 (TGCAAACT TGTCGGGAGGAG, G A C A T G G T C T T C C A C C T C T C T G), EpCAM (TGTCATTTGCTCAAAGCTGGC, C C C C T T C A G T T T T G C T C T T C), CDK1 ( CT T GGCT TCA A ACTGGCTC, T G G TA G A T C C C G G C T TA T TAT T C C), CCNB1 (TGTGGATGCAGAAGATGGAGC, T GACTG T T GC T C T TCCTCAAG) and HPRT 1 (CCCTGGCGTCGTGAT TAGTG, ACACCCTTTCCAAATCCTCAGC) with KAPA SYBR® FAST qPCR Master Mix (2x) Universal Kit (KAPA Biosystems, USA) on a Mastercycler ep realplex machine (Eppendorf, Germany).

Each PCR reaction was performed in duplicate and include $1 \mu 1$ of cDNA, $5 \mu 1$ KAPA SYBR ${ }^{\circledR}$ FAST qPCR Master Mix (2x) Universal Kit (KAPA Biosystems, USA), $1 \mu 1$ of each primer and $2 \mu 1$ water. The following PCR program was used: $95^{\circ} \mathrm{C}$ for $3 \mathrm{~min}, 40$ cycles of $95^{\circ} \mathrm{C}$ for 5 $\mathrm{s}, 30 \mathrm{~s}$ annealing and extension at $60^{\circ} \mathrm{C}$. The equation $2^{-\Delta \mathrm{Ct}}$ was applied to calculate the expression of studied genes, where $\Delta \mathrm{Ct}=\mathrm{Ct}$ of the target gene $-\mathrm{Ct}$ the reference gene (HPRT1). Results are expressed as the number of target gene mRNA copies per 1000 copies of HPRT1 mRNA.

\section{Statistical analysis}

The data was expressed as a mean $\pm \mathrm{SD}$. A ShapiroWilk test was performed to assess the normality of the

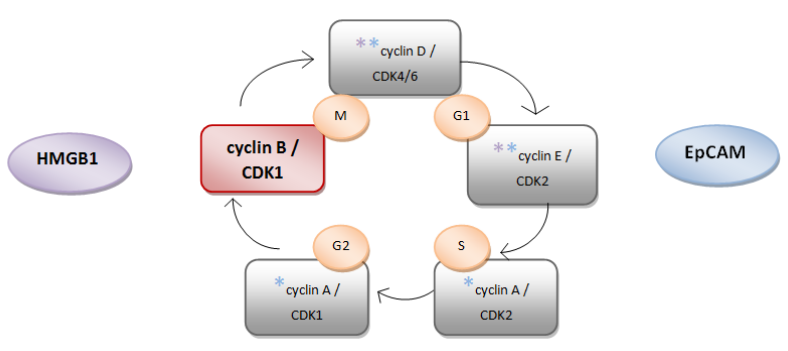

Figure 1. Roles of Cyclin B, CDK1, EpCAM, and HMGB1 in Cell Cycle Progression. Cyclin B / CDK1 controls transition from $\mathrm{G} 2$ to M phase. EpCAM, via affecting cyclins D, E, and A, regulates G1/S and S/G2 progression (*), as HMGB1, through the impact on cyclins D and E, controls only $\mathrm{G} 1 / \mathrm{S}$ progression (*). 
Barbara Bukowska et al

distribution of obtained results. Homogeneity of variance was estimated using the Brown-Forsythe test. An analysis of ANOVA variance with a Tukey post hoc test was used for multiple comparisons. All statistics were calculated by means of the STATISTICA program (StatSoft, Tulsa, OK, USA). A level of significance less than $0.05(\mathrm{p}<0.05)$ was considered significant.

\section{Results}

\section{Cell cycle distribution}

Figures 2 and 3 present the effect of WP 631, Epo B and WP $631+$ Epo B on SKOV-3 cell cycle phase distribution. The cells were treated with drugs for 4,16 or $24 \mathrm{~h}$ and analyzed by flow cytometry. The quantitative results are shown in Figure 2 and the representative histograms of DNA cellular content in SKOV-3 cells are in Figure 3. Figure 2A shows a marked decrease in the number of the
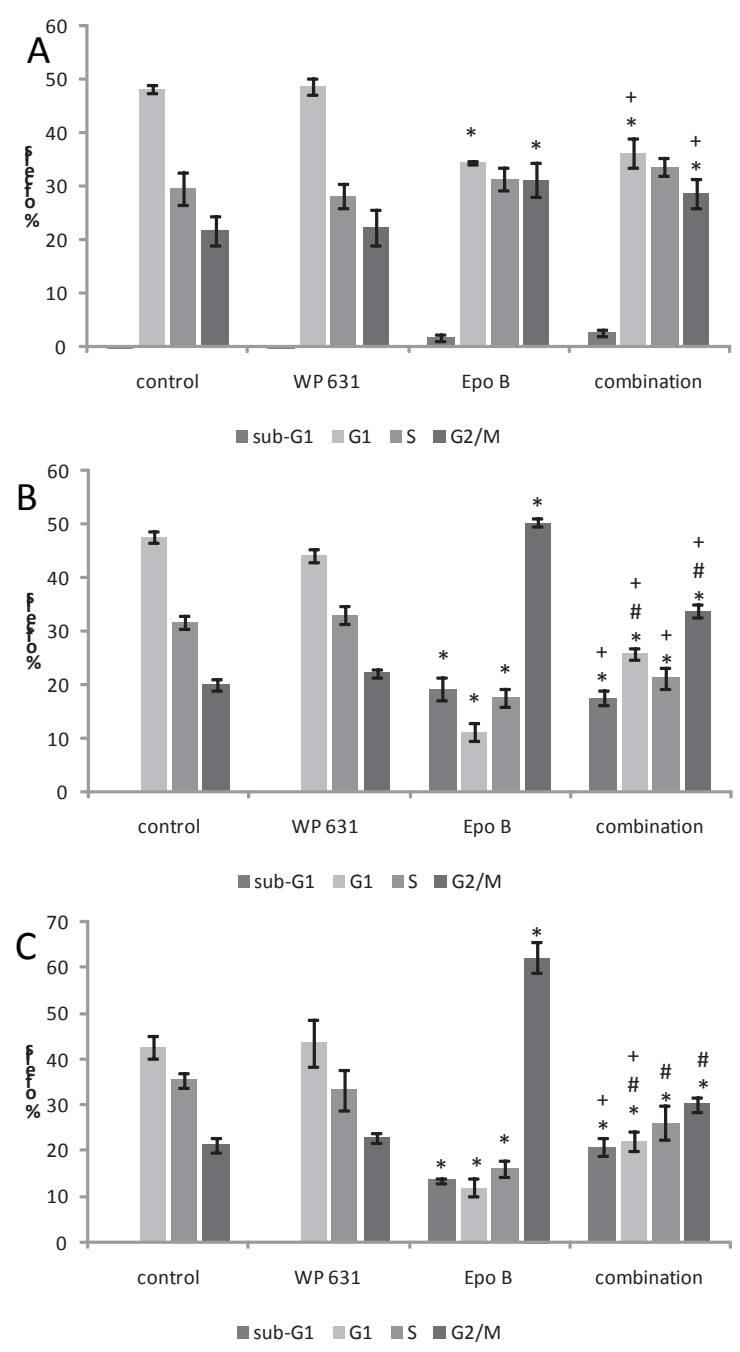

Figure 2. Cell cycle phase distribution in SKOV-3 Cells after $4 \mathrm{~h}(\mathrm{~A}), 16 \mathrm{~h}(\mathrm{~B})$ and $24 \mathrm{~h}(\mathrm{C})$ Incubation with the Drugs. Results are presented as the mean of three experiments \pm SD. (*) Statistically significant differences in comparison to control cells $(\mathrm{p}<0.05)$; (\#) Statistically significant changes noted between the drug combination and Epo B $(\mathrm{p}<0.05)$; $(+)$ Statistically significant changes noted between the drug combination and WP $631(\mathrm{p}<0.05)$
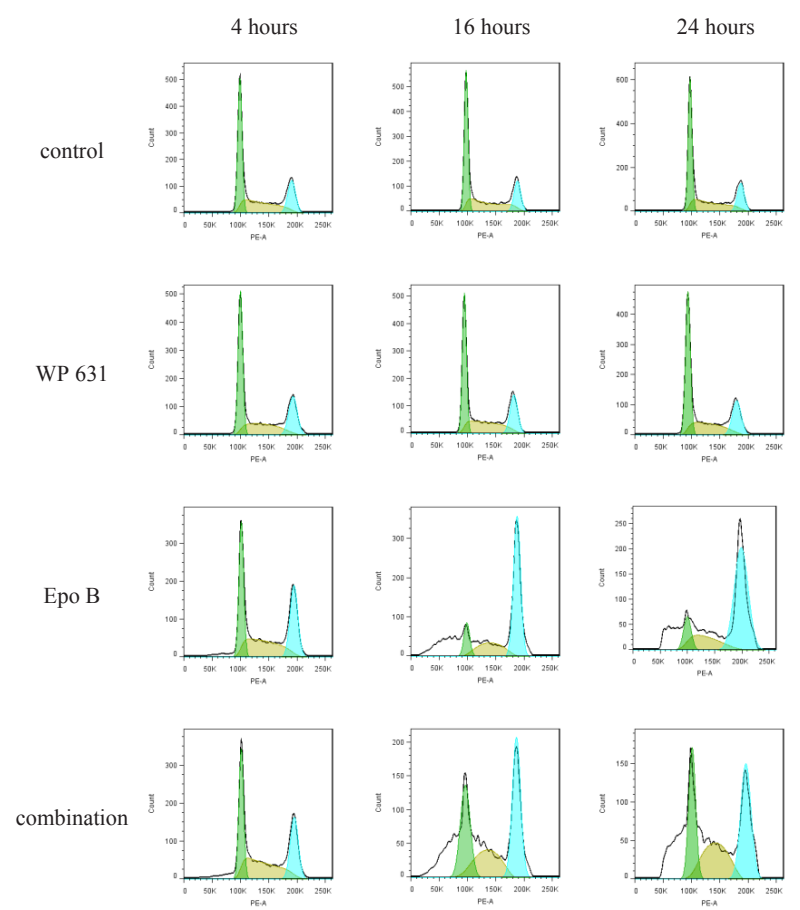

Figure 3. Representative Histograms of SKOV-3 DNA Content after Treatment with WP 631, Epo B or a Combination for 4, 16, and 24 hours
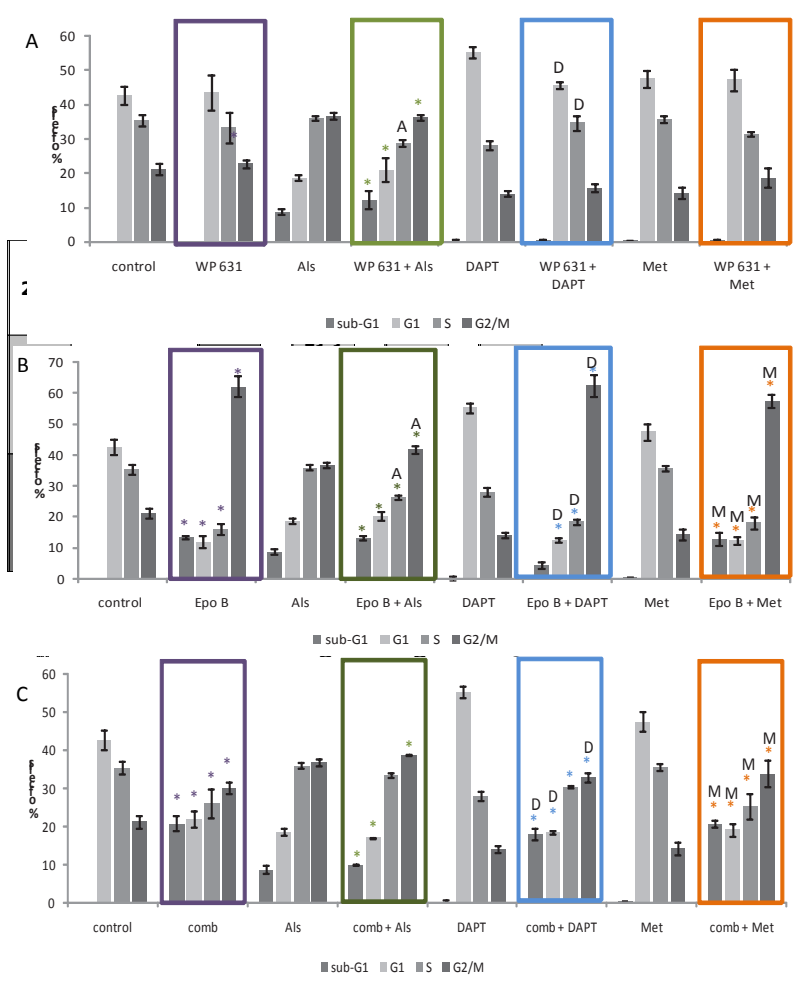

Figure 4. The Influence of WP 631 (A), Epo B (B) and the Drug Combination (C) on Cell Cycle Distribution after 1 Hour Preincubation with Alsterpaullone, DAPT and Metformin. Als - alsterpaullone, Met - metformin. The results are presented as the mean of three experiments \pm SD. $(*)$ Statistically significant changes between drug or a combination of inhibitor + drug and untreated control cells $(p<0.05)$. The letters: A, D, M present statistically significant changes between a combination of inhibitor and drug in comparison to cells treated only with alsterpaullone, DAPT and metformin, respectively $(\mathrm{p}<0.05)$ 
Why a Combination of WP 631 and Epo B is an Improvement on the Drugs Singly - Involvement in the Cell Cycle and Mitotic Slippage

cells in $\mathrm{G} 1$ phase at $4 \mathrm{~h}$ incubation after treatment with Epo B and the drug combination. Moreover, a significant rise in cells arrested in the $\mathrm{G} 2 / \mathrm{M}$ phase was observed. WP 631 , in the tested concentration, did not cause changes in any measured phases. After $16 \mathrm{~h}$ (Figure 2B), Epo B and the drug combination treatment increased the number of cells in the $\mathrm{G} 2 / \mathrm{M}$ phase from $20.0 \pm 1.15 \%$ (control) to $50.33 \pm 0.82 \%$ (Epo B) and $33.82 \pm 1.16 \%$ (combination). Noteworthy is the fact that the drug combination-induced cell cycle arrest was not as high as after Epo B. After this time, the number of apoptotic cells (sub-G1 phase) was at the level of about $20 \%$ after Epo B and drug combination treatment. After $24 \mathrm{~h}$ incubation (Figure 2C), Epo B induced the highest G2/M arrest (a drop from $21.29 \pm 1.68 \%$ for control cells to $62.28 \pm 3.43 \%$ for Epo B-treated ones) and also a rise in sub-G1 population to about $13 \%$. At this point of time, the combination of drugs also stopped the cell cycle at the G2/M phase, but the percentage of cells was lower in comparison to Epo $\mathrm{B}(30.13 \pm 1.58 \%)$. The drug combination in turn caused a stronger apoptosis, observed as a more significant growth of sub-G1 cells (about 21\%). It is worth noting that WP 631 at this concentration did not influence on the SKOV-3 cell cycle in any time.

To summarize, Epo B and the combination of Epo B and WP 631 induced apoptosis, most significantly after treatment with the drug combination, at 16 and $24 \mathrm{~h}$. The fraction of G2/M cells after treatment with Epo B increased in a time-dependant manner, whereas the drug combination resulted in an increase of mitotic cell arrest at the level of about $30 \%$.
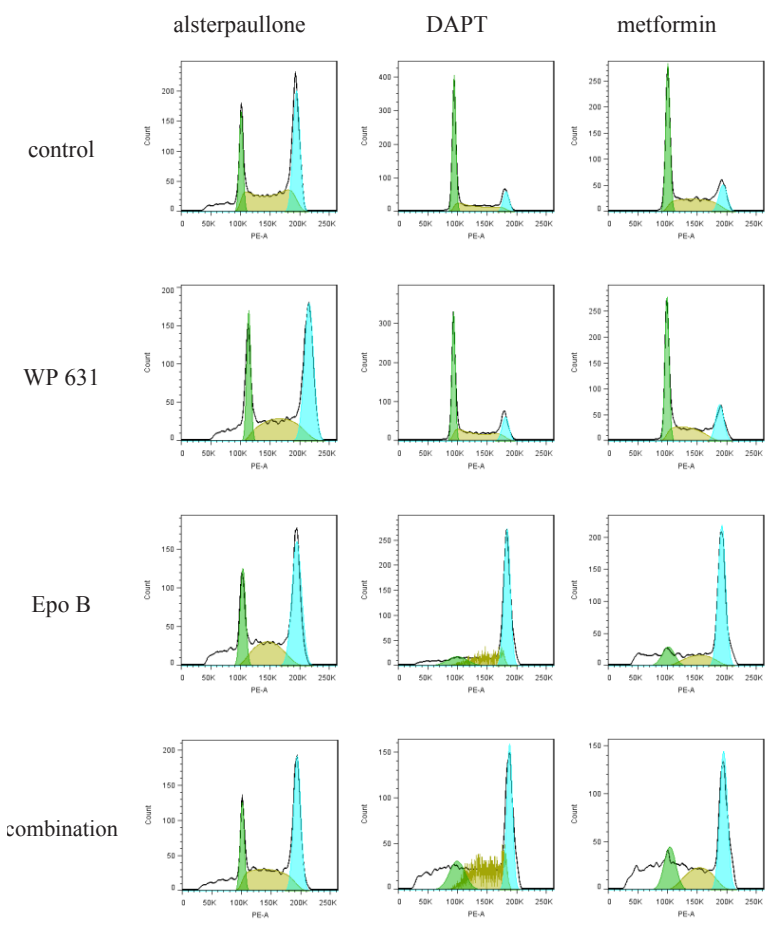

Figure 5. Representative histograms of SKOV-3 DNA content after 1 hour pretreatment with alsterpaullone, DAPT or metformin, and then appropriate treatment with WP 631, Epo B or a combination for 24 hours
The effect of WP 631, Epo B and the drug combination on cell cycle - regulating proteins

SKOV-3 cells were preincubated with alsterpaullone $(10 \mu \mathrm{M})$, DAPT $(50 \mu \mathrm{M})$ or metformin $(5 \mathrm{mM})$ for 1 hour, which inhibited the following cell cycle-regulating proteins: CDK1/cyclin B complex, EpCAM and HMGB1, respectively. The results are presented at Figure 4 (quantitative data) and Figure 5 (histograms).

As we can see in Figure 4A, DAPT and metformin
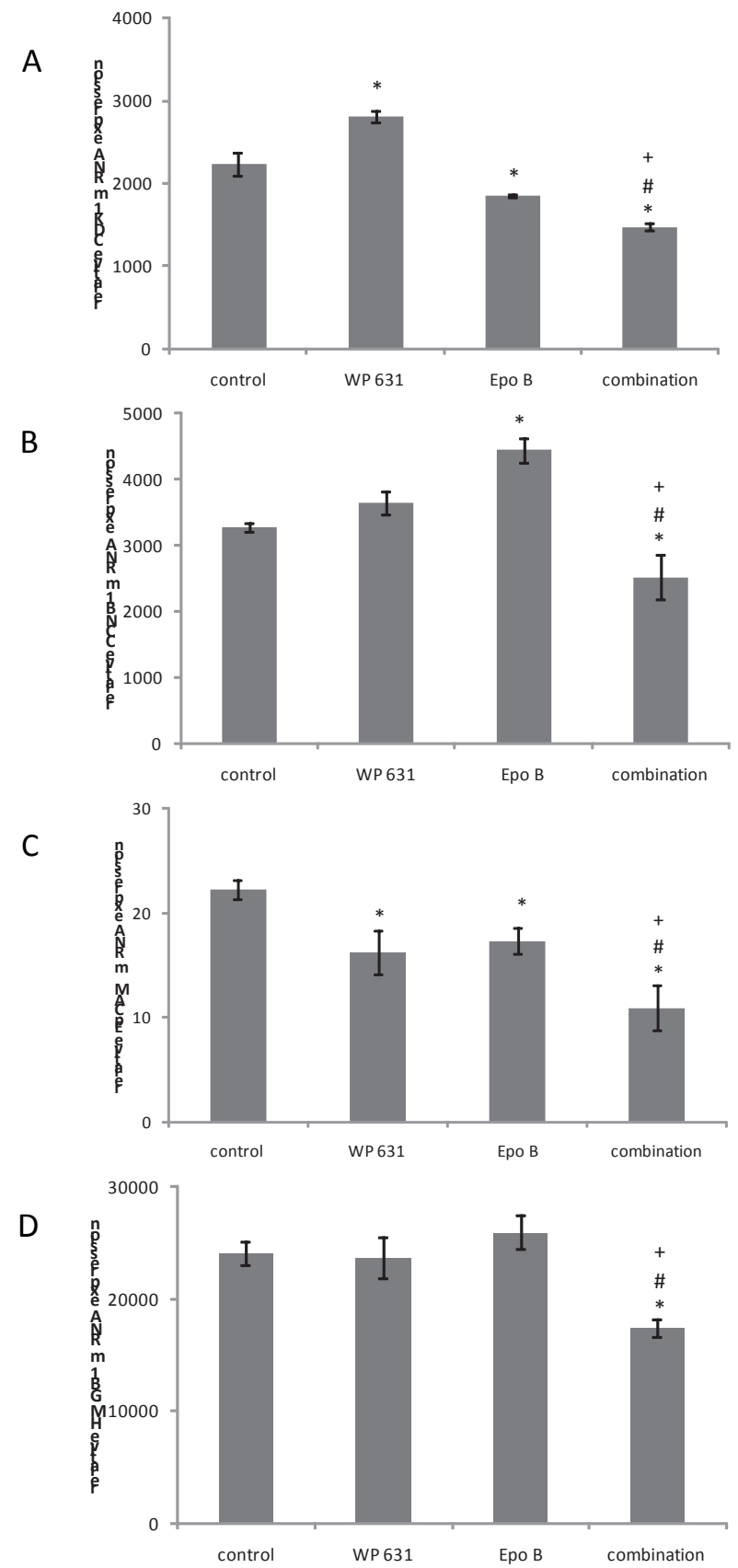

Figure 6. Relative CDK1 (A), CCNB1 (B), EpCAM (C) and HMGB1 (D) mRNAs Expression in Drug-treated and Untreated SKOV-3 Ovarian Cancer Cells after 24

h. Results are presented as the mean of three experiments \pm SD . (*) Statistically significant differences in comparison to control cells ( $\mathrm{p}<0.05)$; (\#) Statistically significant changes noted between the drug combination and Epo B $(\mathrm{p}<0.05) ;(+)$ Statistically significant changes noted between the drug combination and WP $631(\mathrm{p}<0.05)$ 


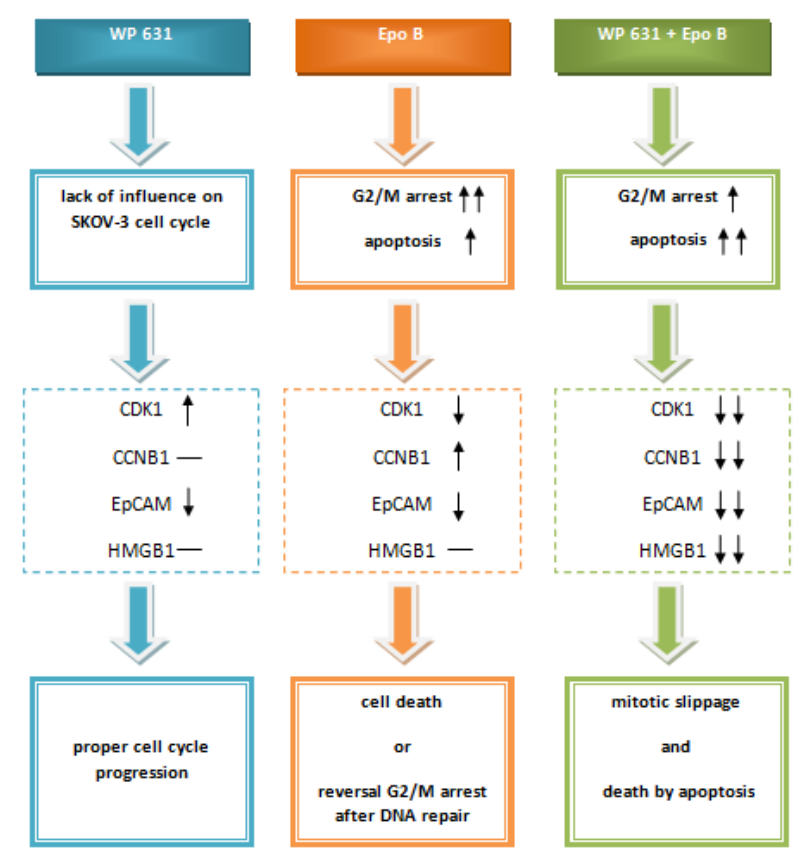

Figure 7.Summary of the effects of the tested drugs on the SKOV-3 cell cycle and the consequences ofChanges for Cancer Cell Viability or Death

did not change the activity of WP 631 . In the probes preincubated with alsterpaullone, a rapid growth of the sub-G1 population $(12.33 \pm 2.71 \%)$ was noticed, as well as an increase of cells in $\mathrm{G} 2 / \mathrm{M}$ phase (from $22.78 \pm 1.16 \%$ to $36.34 \pm 0.82 \%$ ) and a drop in the number of G1 cells (from $43.60 \pm 5.25 \%$ to $21.07 \pm 3.60 \%$ ). Comparing the sections of the graph for alsterpaullone, WP 631 and alsterpaullone + WP 631, we can definitely predict that the compound responsible for the observed changes in cell cycle distribution is an inhibitor, not a bisanthracycline. This observation pointed to the fact that the activity of WP 631 does not depend on CDK1/cyclin B complex.

Figure 4B presents differences in Epo B activity after using cell cycle inhibitors. From all tested compounds, only alsterpaullone changed the influence of Epo B on the SKOV-3 cell cycle. Preincubation with CDK1/cyclin $\mathrm{B}$ inhibitor caused a significant drop in the level of cells arrested at $\mathrm{G} 2 / \mathrm{M}$ phase (from $62.28 \pm 3.43 \%$ to 41.80 $\pm 1.39 \%$ ). G2/M arrest was not completely extinguished after pretreatment with alsterpaullone, which testifies to the fact that CDK1/cyclin B complex is not the only protein mediating Epo B activity.

Figure 4C shows the cell cycle distribution after treatment with the drug combination preceded by 1-hour preincubation with alsterpaullone, DAPT or metformin. The latter two compounds did not affect regimen activity. Preincubation with alsterpaullone caused a marked decrease in the level of sub-G1 $(9.96 \pm 0.22 \%)$ cells in comparison to cells treated only with WP $631+$ Epo B $(20.81 \pm 1.97 \%)$. Additionally, a rise in the number of cells arrested at the $\mathrm{G} 2 / \mathrm{M}$ phase was observed (from $30.13 \pm 1.58 \%$ to $38.84 \pm 0.28 \%$ ). No significant changes between cells incubated with alsterpaullone and cells exposed to alsterpaullone and then the drug combination were noticed, demonstrating that Epo B in lower concentration used in a combination $(5 \mathrm{nM})$ exhibits its activity through more distinct mechanisms than Epo B used at the concentration of $10 \mathrm{nM}$.

CDK1, cyclin B, EpCAM and HMGB1 mRNAs expression after treatment of SKOV-3 cells with WP 631, Epo $B$ and a combination of the drugs

Figure 6A presents the expression of CDK1 mRNA in drug-treated and untreated SKOV-3 cells after $24 \mathrm{~h}$. Among all the used compounds, only WP 631 caused an increase in the level of CDK1 mRNA expression, whereas Epo B and the drug combination manifested the opposite effects. The influence of WP 631, Epo B and their combination on cyclin B (CCNB1) mRNA expression is shown in Figure 6B. Treatment with Epo B resulted in a rise of CCNB1 mRNA expression, while the combination decreased it. As can be seen in Figure 6C, WP 631 and Epo $\mathrm{B}$, as well as their combination led to a fall of EpCAM mRNA expression. Interestingly, the mRNA level showed the most significant decrease after drug regimen treatment. Only the combined action of WP 631 and Epo B influenced HMGB1 mRNA expression, leading to its drop, which is presented in Figure 6 D.

\section{Discussion}

The presented data confirm and expand our previous studies, in which the synergism between WP 631 and Epo B was well established (Marczak et al., 2014; Rogalska et al., 2014). The main aim of this research was to explain the reasons for the higher toxicity of the drug combination in comparison to the effect of the single agents. We supposed that cell cycle-regulating proteins may be involved in the mechanism of the action of the drug combination. It was also believed that evaluation of the sub-G1 population would reveal a correlation between apoptosis and cell cycle distribution. In order to check our hypothesis, we decided to investigate the effect of WP 631 and Epo B used together on the cell cycle of SKOV-3.

Literature data provide a wide spectrum of information regarding the disturbances of the cell cycle of cancer cells after administration of Epo B as well as WP 631. Both of the tested drugs induced cell cycle arrest at G2/M phase (Pozarowski et al., 2004; Mansilla et al., 2006; Lee et al., 2007; Pellicciotta et al., 2013). It should be highlighted that in the cited studies, bisanthracycline was used at much higher concentrations than in our experiments, which explains the lack of the influence of $10 \mathrm{nM} \mathrm{WP} 631$ on cell cycle distribution at all tested times in our experiments. In turn, results for Epo B are in compliance with literature data: the drug caused time-dependent growth of cells arrested at G2/M. It is supposed that after such prolonged arrest, the cells usually die via apoptosis during mitotic arrest (Orth et al., 2012).

However, we feel most interesting are our results obtained for the drug combination. It was noticed that Epo B administered in combination with WP 631 had a lower ability to induce the stoppage of the cell cycle than Epo B given alone. It may be speculated that after exposure of the cells to antimitotic drugs (including Epo B), the cells may escape from mitotic arrest and enter the 

next $\mathrm{G} 1$ phase with decodensated chromosomes. The cells that exit mitosis do not undergo cell division and become tetraploid. This process is known as mitotic slippage. The latter phenomenon enables the cells to transit to the G1 phase without DNA division, even though the spindle is damaged. In the cells that exited mitosis, "tetraploidy checkpoint" assesses DNA content and then triggers apoptosis (Blagosklonny, 2007; Endo et al., 2010; Asraf et al., 2015). Apoptosis is induced on two levels. In Epo B-treated cells, apoptosis appears indirectly during mitotic arrest, whereas in combination-treated cells after mitotic slippage. However, apoptosis can also occur independently of cell cycle disturbances under certain circumstances, including ROS (reactive oxygen species) generation or DNA damage (Lupertz et al., 2010). As we have shown in our earlier studies, the drug combination caused a higher increase in ROS production than the single drugs as well as the most significant DNA damage (Rogalska et al., 2014). In turn, our current studies revealed that the drug combination induced the highest growth in sub-G1cells among all tested substances. These observations give us a new insight into the mechanism of the action of the WP 631 and Epo B combination. As apoptosis could be induced independently of cell cycle arrest, it may explain the higher toxicity of the drug regimen in comparison to single agents.

One of the major factors required for mitotic slippage is the degradation of cyclin $\mathrm{B}$. We have shown that the combination of WP 631 and Epo B caused a marked decrease in the level of CCNB1 mRNA expression, which is evidence of cyclin B destruction. In contrast, Epo B increased CCNB1 expression preventing Epo B-treated cells escaping from mitotic arrest (Figure7). Although literature data does provide some information that Epo B may cause mitotic slippage at the concentration of $40 \mathrm{nM}$ (Chen et al., 2003), it does not provide any information about WP 631-caused mitosis exit.

We have established that the drug combination leads to mitotic slippage. But it also must be explained how the combination-treated cells die after mitotic exit. The most likely possibility is mitotic slippage, followed by cell death through apoptosis (Ye et al., 2014; Asraf et al., 2015). Our previous studies confirmed the potent ability of the drug combination to induce apoptosis, much higher than the capacities of the single drugs (Marczak et al., 2014; Rogalska et al., 2014). Taking all these indications into account, the obtained results are highly advantageous. The cells exposed to the drug combination were, to lesser extent, stopped at G2/M phase, suggesting that they had undergone mitotic slippage. After exiting mitosis, it is mainly apoptosis that is responsible for their death. On the other hand, the G2/M arrest caused by Epo B may be reversible. The results of Risinger and Moobery confirmed that paclitaxel and other microtubule stabilizers induced reversible G2/M arrest in HeLa cells (Risinger and Mooberry, 2011). Moreover, G2/M arrest caused by an antimitotic agent, nocodazole, with the ability to prevent microtubule polymerization, was reversed in Hydra cells (Buzgariu et al., 2014). In our previous article, we have shown that the DNA damage induced by Epo B are repaired to a great degree (Rogalska et al., 2014).
In contrast, apoptosis which mainly occurs after mitotic slippage is believed to be irreversible (Tang et al., 2012). This highly effective DNA repair system in Epo B-treated cells, as well as apoptosis irreversibility, may easily clarify why the drug combination has a higher activity and constitutes a better therapeutic option in comparison to Epo B alone.

Our observations indicate that Epo B by itself at a concentration of $10 \mathrm{nM}$ led to mitotic arrest and apoptosis, whereas the drug combination (with Epo B at a dose of $5 \mathrm{nM}$ ) caused mitotic slippage, which agrees with the statement of Mukhtar et al., who also pointed out that low concentrations of microtubule stabilizers cause an exit from mitosis, while higher drug dosages lead to mitotic block in which cells die via apoptosis (Mukhtar et al., 2014).

The purpose of our continued studies was to find cell cycle-related proteins which contribute to the drugs' activity. In order to achieve this, the activities of CDK1/cyclin B, EpCAM and HMGB1 were blocked by appropriate inhibitors (alsterpaullone, DAPT and metformin, respectively) and then the cell cycle distribution was evaluated. WP 631 at applied concentration of $10 \mathrm{nM}$ did not influence the cell cycle of SKOV-3 cells. It was easy to predict that the inhibition of CDK1, cyclin B, EpCAM or HMGB1 would not bring significant changes in WP 631-treated cells. The growth of the sub-G1 fraction, as well as G2/M arrest, were observed only after CDK1/cyclin B inhibition, but, with absolute certainty, it was the effect of alsterpaullone which exhibits proapoptotic properties (Cui et al., 2013).

The usage of the inhibitors enabled us to confirm that Epo B acts via the CDK1/cyclin B complex. As proof, we offer the observation that inhibition of CDK1/cyclin $\mathrm{B}$ complex is responsible for a decrease in Epo B activity, observed as a marked drop in cells arrested at the G2/M phase. However, the level of G2/M cells was not reduced to control values, which we believe points to the fact that other mechanisms are engaged in the Epo B activity. We have also noticed, for the first time, that Epo B decreases the mRNA level of CDK1 (Figure 7), testifying that Epo $\mathrm{B}$ itself may also act as a CDK1 inhibitor which runs contrary to the results of Yang et al., who focused on the activity of MJ-29, a compound with the ability to disrupt microtubule organization, and establish an increase in CDK1 level (Yang et al., 2010). However, in recent years, many therapeutic approaches based on CDK inhibition have been developed, indicating that it may be a promising strategy for the treatment of ovarian cancer (Chen et al., 2015a; Chen et al., 2015b), meaning that Epo B may bring a dual benefit, as a microtubule stabilizer as well as a CDK1 inhibitor.

Although we have demonstrated that Epo B exhibits its activity via CDK1/cyclin B-mediated pathway, this observation was not confirmed in the case of the drug combination. Preincubation with alsterpaullone resulted in an increase of G2/M-arrested cells, as well as a drop in sub-G1 population. Firstly, those results suggest that the drug combination has another mechanism of action than Epo B alone and, to a lesser extent, indirectly affects CDK1/cyclin B complex. It could be explained by the 
antagonistic action of WP 631 and Epo B on CDK1 activity. As mentioned above, anthracyclines and taxanes exhibit opposite effects on this kinase (Nakayama et al., 2009). In addition, we have noticed that the drug combination caused a greater decrease of cyclin B and CDK1 mRNAs expression (Figure 7). As described above, degradation of cyclin $\mathrm{B}$ is responsible for mitotic slippage, which is probably induced by the drug combination. It may be speculated that prolonged mitotic block may contribute to the inactivation of CDK1, due to the slow cyclin B depletion, thus leading to mitotic exit (Brito and Rieder, 2006; Riffell et al., 2011). It is consistent with our observation that the drug combination, after which mitotic slippage occurs, decreases CDK1 and cyclin B expression. It was also noticed that preincubation with alsterpaullone significantly decreases sub-G1 fraction (in comparison to combination-treated cells). McClendon et al. pointed out that CDK4/6 inhibitors protect RBproficient breast cancer cells from doxorubicin-mediated toxicity, resulting in the preservation of cell viability in the presence of anthracycline (McClendon et al., 2012). Taking this into account, the drop in the level of sub-G1 cells could be possibly caused by the antagonism between CDK inhibitor and WP 631.

The results of EpCAM and HMGB1 expression after drug treatment are highly attractive. For a review see Figure 7. We have indicated that the combination of WP 631 and Epo B significantly decreases EpCAM mRNA expression in SKOV-3 cells more than single agents. Shahabi et al. demonstrated that Epo B enhances surface expression of EpCAM, while not influencing EpCAM mRNA expression (Shahabi et al., 2010). There are no available data about the effects of WP 631 on EpCAM, which is overexpressed in most human carcinomas, including ovarian cancer. The precise role of EpCAM in cancer growth and metastasis remains elusive. Its overexpression correlates with a decreased survival rate in patients at stage III/IV of ovarian carcinomas (Nunna et al., 2014). It is also a poor prognostic factor for many others cancers, breast cancer being one of them (Soysal et al., 2013). EpCAM overexpression may serve as a therapeutic target, for example catumaxomab, an antibody directed against EpCAM, received the approval of EMA (European Medicines Agency) for ovarian cancer treatment (Tse et al., 2014). When EpCAM is downregulated, it suppresses the metastasis and proliferation of cancer cells $(\mathrm{Mu}$ et al., 2014). The WP 631 + Epo B combination led to a significant drop in the level of EpCAM mRNA.

HMGB1 is a recently identified protein that is overexpressed in ovarian cancer and responsible for poor pathological features (Li et al., 2015). Chen et al. indicated that HMGB1 overexpression correlated with elevated invasiveness and inhibited apoptosis in the SKOV-3 line. Thus, knockdown of HMGB1 resulted in a suppression of ovarian cancer cell proliferation and apoptosis induction. Levels of the serum HMGB1 were far higher in patients with advanced cancers, suggesting that a high level of HMGB 1 is associated with advanced disease grade and, thus, with less chance for a cure (Chen et al., 2012). Furthermore, HMGB1 overexpression may affect effective anticancer therapy through stimulating drug resistance development (Ohmori et al., 2015). For these reasons, decreasing HMGB1 expression is highly desirable, improving disease outcome in patients with ovarian cancer. We have demonstrated that the drug combination leads to a significant drop in the level of HMGB1 mRNA, whereas WP 631 and Epo B applied separately did not influence HMGB1 level. It is another premise explaining the higher toxicity of the drug combination in comparison to single drugs.

In summary, Epo B and the combination of WP 631 and Epo B disturb the cell cycle in SKOV-3 cells. The combination of WP 631 and Epo B leads to lower G2/M arrest than single Epo B. We suggest that the drug combination-treated cells exited mitotic arrest, known as mitotic slippage, and then died in apoptosis. This observation is highly beneficial, since Epo B-induced cell cycle stoppage may be reversible, while apoptosis, which mainly occurs after mitotic slippage, cannot be overturned. Our further studies allow us to state that Epo B acts via CDK1/cyclin B - mediated pathway and has the ability to indirectly inhibit CDK1. Unfortunately, those findings were not confirmed for the drug combination, suggesting firstly that the regimen affects another cell cycle-regulating protein and secondly, that alsterpaullone, a CDK inhibitor, possibly antagonizes the activity of WP 631 . We have also established that the combined action of WP 631 and Epo B leads to a marked decrease in the level of EpCAM and HMGB 1 expression, which translates into a higher toxicity of the drug combination and the suppression of cell proliferation. These two proteins serve as prognostic markers, whose overexpression relates to a poor outcome prognosis and, thus, their downregulation could contribute to therapy improvement. These latest findings regarding the activity of WP 631 and Epo B combination in SKOV3 , even though very promising, require further studies.

\section{References}

Arikan SK, Kasap B, Yetimalar H, et al (2014). Impact of prognostic factors on survival rates in patients with ovarian carcinoma. Asian Pac J Cancer Prev, 15, 6087-94.

Asraf H, Avunie-Masala R, Hershfinkel M, et al (2015). Mitotic slippage and expression of survivin are linked to differential sensitivity of human cancer cell-lines to the Kinesin-5 inhibitor monastrol. PLoS One, 10, 129255.

Blagosklonny MV (2007). Mitotic arrest and cell fate: why and how mitotic inhibition of transcription drives mutually exclusive events. Cell Cycle, 6, 70-4.

Brito DA, Rieder CL (2006). Mitotic checkpoint slippage in humans occurs via cyclin B destruction in the presence of an active checkpoint. Curr Biol, 16, 1194-200.

Buzgariu W, Crescenzi M, Galliot B (2014). Robust G2 pausing of adult stem cells in Hydra. Differentiation, 87, 83-99.

Chaves-Perez A, Mack B, Maetzel D, et al (2013). EpCAM regulates cell cycle progression via control of cyclin D1 expression. Oncogene, 32, 641-50.

Chen J, Liu X, Zhang J, et al (2012). Targeting HMGB1 inhibits ovarian cancer growth and metastasis by lentivirus-mediated RNA interference. J Cell Physiol, 227, 3629-38.

Chen JG, Yang CP, Cammer M, et al (2003). Gene expression and mitotic exit induced by microtubule-stabilizing drugs. Cancer Res, 63, 7891-9. 
Why a Combination of WP 631 and Epo B is an Improvement on the Drugs Singly - Involvement in the Cell Cycle and Mitotic Slippage

Chen S, Chen X, Xiu YL, et al (2015a). MicroRNA-490-3P targets CDK1 and inhibits ovarian epithelial carcinoma tumorigenesis and progression. Cancer Lett, 362, 122-30.

Chen XX, Xie FF, Zhu XJ, et al (2015b). Cyclin-dependent kinase inhibitor dinaciclib potently synergizes with cisplatin in preclinical models of ovarian cancer. Oncotarget, 6, 14926-39.

Chen Y, Liu ZJ, Liu J, et al (2014). Inhibition of metastasis and invasion of ovarian cancer cells by crude polysaccharides from rosa roxburghii tratt in vitro. Asian Pac J Cancer Prev, 15, 10351-4.

Chow JP, Poon RY, Ma HT (2011). Inhibitory phosphorylation of cyclin-dependent kinase 1 as a compensatory mechanism for mitosis exit. Mol Cell Biol, 31, 1478-91.

Chuang P-Y, Huang C, Huang H-C (2013). The use of a combination of tamoxifen and doxorubicin synergistically to induce cell cycle arrest in BT483 cells by down-regulating CDK1, CDK2 and cyclin D expression. J Pharmaceutical Technol Drug Res, $2,12$.

Cui C, Wang Y, Wang Y, et al (2013). Alsterpaullone, a CyclinDependent Kinase Inhibitor, Mediated Toxicity in HeLa Cells through Apoptosis-Inducing Effect. J Anal Methods Chem, 2013, 602091.

Endo K, Mizuguchi M, Harata A, et al (2010). Nocodazole induces mitotic cell death with apoptotic-like features in Saccharomyces cerevisiae. FEBS Lett, 584, 2387-92.

Enserink JM, Kolodner RD (2010). An overview of Cdk1controlled targets and processes. Cell Div, $\mathbf{5}, 11$.

Gong H, Zuliani P, Komuravelli A, et al (2010). Analysis and verification of the HMGB1 signaling pathway. $B M C$ Bioinformatics, 11, 10.

Griffin D, Wittmann S, Guo F, et al (2003). Molecular determinants of epothilone B derivative (BMS 247550) and Apo-2L/TRAIL-induced apoptosis of human ovarian cancer cells. Gynecol Oncol, 89, 37-47.

Jiao Y, Wang HC, Fan SJ (2007). Growth suppression and radiosensitivity increase by HMGB1 in breast cancer. Acta Pharmacol Sin, 28, 1957-67.

Kobayashi H, Saito T, Sato K, et al (2014). Phosphorylation of cyclin-dependent kinase 5 (Cdk5) at Tyr-15 is inhibited by $\mathrm{Cdk} 5$ activators and does not contribute to the activation of Cdk5. J Biol Chem, 289, 19627-36.

Lee SH, Son SM, Son DJ, et al (2007). Epothilones induce human colon cancer SW620 cell apoptosis via the tubulin polymerization independent activation of the nuclear factorkappaB/IkappaB kinase signal pathway. Mol Cancer Ther, 6, 2786-97.

Li H, Huang W, Luo R (2015). The microRNA-325 inhibits hepatocellular carcinoma progression by targeting high mobility group box 1. Diagn Pathol, 10, 117.

Lin CW, Liao MY, Lin WW, et al (2012). Epithelial cell adhesion molecule regulates tumor initiation and tumorigenesis via activating reprogramming factors and epithelialmesenchymal transition gene expression in colon cancer. $J$ Biol Chem, 287, 39449-59.

Lupertz R, Watjen W, Kahl R, et al (2010). Dose- and timedependent effects of doxorubicin on cytotoxicity, cell cycle and apoptotic cell death in human colon cancer cells. Toxicol, 271, 115-21.

Mansilla S, Priebe W, Portugal J (2006). Mitotic catastrophe results in cell death by caspase-dependent and caspaseindependent mechanisms. Cell Cycle, 5, 53-60.

Marczak A, Bukowska B, Rogalska A (2014). WP 631 and Epo B synergize in SKOV-3 human ovarian cancer cells. Environ Toxicol Pharmacol, 37, 256-66.

McClendon AK, Dean JL, Rivadeneira DB, et al (2012). CDK4/6 inhibition antagonizes the cytotoxic response to anthracycline therapy. Cell Cycle, 11, 2747-55.

Mu Y, Sa N, Yu L, et al (2014). Epithelial cell adhesion molecule is overexpressed in hypopharyngeal carcinoma and suppresses the metastasis and proliferation of the disease when downregulated. Oncol Lett, 8, 175-82.

Mukhtar E, Adhami VM, Mukhtar H (2014). Targeting microtubules by natural agents for cancer therapy. Mol Cancer Ther, 13, 275-84.

Munz M, Kieu C, Mack B, et al (2004). The carcinomaassociated antigen EpCAM upregulates c-myc and induces cell proliferation. Oncogene, 23, 5748-58.

Nakayama S, Torikoshi Y, Takahashi T, et al (2009). Prediction of paclitaxel sensitivity by CDK1 and CDK2 activity in human breast cancer cells. Breast Cancer Res, 11, 12.

Nunna S, Reinhardt R, Ragozin S, et al (2014). Targeted methylation of the epithelial cell adhesion molecule (EpCAM) promoter to silence its expression in ovarian cancer cells. PLoS One, 9, 87703.

Ohmori H, Yi L, Fujii K, Sasaki T, Kuniyasu H (2015). High mobility group box 1 induces cancer aggressiveness and drug resistance. Ann Clin Pathol, 3, 1042.

Orth JD, Loewer A, Lahav G, et al (2012). Prolonged mitotic arrest triggers partial activation of apoptosis, resulting in DNA damage and 553 induction. Mol Biol Cell, 23, 567-76.

Pellicciotta I, Yang CP, Venditti CA, et al (2013). Response to microtubule-interacting agents in primary epithelial ovarian cancer cells. Cancer Cell Int, $\mathbf{1 3}, 33$.

Pozarowski P, Huang X, Gong RW, et al (2004). Simple, semiautomatic assay of cytostatic and cytotoxic effects of antitumor drugs by laser scanning cytometry: effects of the bis-intercalator WP631 on growth and cell cycle of T-24 cells. Cytometry A, 57, 113-9.

Riffell JL, Janicke RU, Roberge M (2011). Caspase-3-dependent mitotic checkpoint inactivation by the small-molecule inducers of mitotic slippage SU6656 and geraldol. Mol Cancer Ther, 10, 839-49.

Risinger AL, Mooberry SL (2011). Cellular studies reveal mechanistic differences between taccalonolide $\mathrm{A}$ and paclitaxel. Cell Cycle, 10, 2162-71.

Rogalska A, Bukowska B, Marczak A (2014). Caspases and ROS - dependent mechanism of action mediated by combination of WP 631 and epothilone B. Anticancer Agents Med Chem, 14, 1261-70.

Saito T, Chiba T, Yuki K, et al (2013). Metformin, a diabetes drug, eliminates tumor-initiating hepatocellular carcinoma cells. PLoS One, $\mathbf{8}, 70010$.

Schnell U, Cirulli V, Giepmans BN (2013). EpCAM: structure and function in health and disease. Biochim Biophys Acta, 1828, 1989-2001.

Senese S, Lo YC, Huang D, et al (2014). Chemical dissection of the cell cycle: probes for cell biology and anti-cancer drug development. Cell Death Dis, 5, 1462.

Shahabi S, Yang CP, Goldberg GL, et al (2010). Epothilone B enhances surface EpCAM expression in ovarian cancer Hey cells. Gynecol Oncol, 119, 345-50.

Soysal SD, Muenst S, Barbie T, et al (2013). EpCAM expression varies significantly and is differentially associated with prognosis in the luminal B HER2(+), basal-like, and HER2 intrinsic subtypes of breast cancer. Br J Cancer, 108, 1480-7.

Tang HL, Tang HM, Mak KH, et al (2012). Cell survival, DNA damage, and oncogenic transformation after a transient and reversible apoptotic response. Mol Biol Cell, 23, 2240-52.

Tse BW, Collins A, Oehler MK, et al (2014). Antibody-based immunotherapy for ovarian cancer: where are we at? Ann Oncol, 25, 322-31.

Tsoyi K, Jang HJ, Nizamutdinova IT, et al (2011). Metformin inhibits HMGB1 release in LPS-treated RAW 264.7 cells 
Barbara Bukowska et al

and increases survival rate of endotoxaemic mice. $\mathrm{Br} J$ Pharmacol, 162, 1498-508.

van der Gun BT, Melchers LJ, Ruiters MH, et al (2010). EpCAM in carcinogenesis: the good, the bad or the ugly. Carcinogenesis, 31, 1913-21.

Villamarin S, Mansilla S, Ferrer-Miralles N, et al (2003). A comparative analysis of the time-dependent antiproliferative effects of daunorubicin and WP631. Eur J Biochem, 270, 764-70.

Yang JS, Hour MJ, Huang WW, et al (2010). MJ-29 inhibits tubulin polymerization, induces mitotic arrest, and triggers apoptosis via cyclin-dependent kinase 1-mediated Bcl-2 phosphorylation in human leukemia U937 cells.J Pharmacol Exp Ther, 334, 477-88.

Ye H, Karim AA, Loh XJ (2014). Current treatment options and drug delivery systems as potential therapeutic agents for ovarian cancer: a review. Mater Sci Eng C Mater Biol Appl, 45, 609-19.

Zhang T, Hu X, Cai Y, et al (2014). Metformin protects against hyperglycemia-induced cardiomyocytes injury by inhibiting the expressions of receptor for advanced glycation end products and high mobility group box 1 protein. Mol Biol Rep, 41, 1335-40. 